\title{
Robotic Control of a Multi-Modal Rigid Endoscope Combining Optical Imaging with All-Optical Ultrasound
}

\author{
George Dwyer ${ }^{1,2,3}$, Richard J Colchester ${ }^{2,1}$, Erwin J Alles ${ }^{2,1}$, Efthymios Maneas ${ }^{2,1}$, \\ Sebastien Ourselin ${ }^{4}$, Tom Vercauteren ${ }^{4}$, Jan Deprest ${ }^{5}$, Emmanuel Vander Poorten ${ }^{6}$, \\ Paolo De Coppi ${ }^{7,1}$, Adrien E Desjardins ${ }^{2,1}$ and Danail Stoyanov ${ }^{1,8,3}$
}

\begin{abstract}
Fetoscopy is a technically challenging surgery, due to the dynamic environment and low diameter endoscopes often resulting in a limited field of view. In this paper, we report on the design and operation of a robotic multimodal endoscope with optical ultrasound and white light stereo camera. The manufacture and control of the endoscope is presented, along with large area $(80 \mathbf{m m} \times 80 \mathbf{m m})$ surface visualisations of a placenta phantom using the optical ultrasound sensor. The repeatability of the surface visualisations was found to be $0.446 \pm 0.139 \mathrm{~mm}$ and $0.267 \pm 0.017 \mathbf{m m}$ for a raster and spiral scan, respectively.
\end{abstract}

\section{INTRODUCTION}

Many surgical procedures are now being performed minimally invasively, with the use of miniaturised devices, such as catheters and endoscopes. One such procedure is fetoscopy, an established practice within fetal medicine for both diagnostic and therapeutic purposes. By using miniature imaging probes, a number of procedures can be performed during pregnancy minimally-invasively, thereby helping to avoid surgical complications and improving outcomes to both mother and baby. Current instrumentation is based on thin rigid endoscopes comprising working channels that can be used to deliver instrumentation into the environment. One of the main design criteria of these scopes is to minimise the diameter, while also providing the instrumentation channels needed for the procedure [1].

One such minimally-invasive fetal intervention is Fetoscopic Laser Photocoagulation (FLP) which is used to treat Twin-to-Twin Transfusion Syndrome (TTTS). This condition

This work was supported through an Innovative Engineering for Health award by Wellcome Trust [WT101957]; Engineering and Physical Sciences Research Council (EPSRC) [NS/A000027/1], the EPSRC-funded UCL Centre for Doctoral Training in Medical Imaging (EP/L016478/1), ERC Starting Grant [310970 MOPHIM], the EU project FAMOS [FP7 ICT, Contract 317744], the EPSRC Healthcare Technologies Challenge Award [EP/N021177/1], EPSRC grants (EP/N027078/1, EP/P012841/1, EP/P027938/1, EP/R004080/1).

${ }^{1}$ Wellcome / EPSRC Centre for Interventional and Surgical Sciences, University College London (UCL), London, UK

${ }^{2}$ Department of Medical Physics and Biomedical Engineering, UCL, London, UK

${ }^{3}$ UCL Robotics Institute, UCL, London, UK

${ }^{4}$ School of Biomedical Engineering and Imaging Sciences, Kings College London, London, UK

${ }^{5}$ Department of Obstetrics and Gynaecology, University Hospital Leuven, Leuven, Belgium and Department of Obstetrics and Gynaecology, UCL, London, UK

${ }^{6}$ Dept. Mechanical Engineering, KU Leuven, Leuven, Belgium

${ }^{7}$ Institute of Child Health, UCL, London, UK

${ }^{8}$ Department of Computer Science, UCL, London, UK

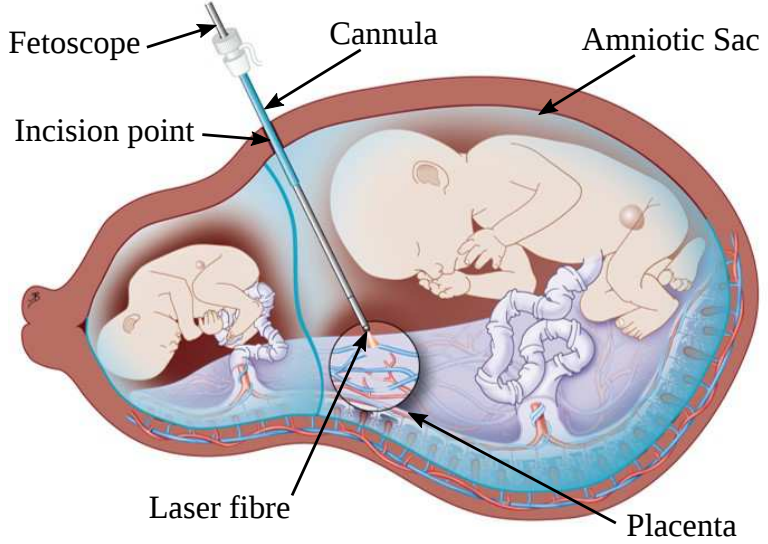

Fig. 1. Schematic of the fetoscopic laser photocoagulation procedure for the treatment of twin-to-twin transfusion syndrome. Image reproduced and modified with permission from UZ Leuven, Belgium.

affects twins sharing a placenta, where an uneven and harmful shared blood supply is formed between the twins across the placenta. In clinical practice, the FLP procedure involves scanning the endoscope across the placenta to identify the anastomoses, which are then coagulated using a laser inserted through the working channel. Clinical guidelines for coagulation is for the laser to be positioned approximately $10 \mathrm{~mm}$ from the vessel and orientated normal to the surface [2].

Due to their small form factor, fetoscopes often suffer from a limited field of view, which complicates the manipulation of these endoscopes [1]. While fetoscopy is traditionally performed manually, incorporating robotics has the potential to improve the stability of imaging devices through precise control of the instrumentation [3], [4]. In addition, the ability to execute pre-computed trajectories and utilise feedback from other sensors, such as strain gauges for force control, can improve image acquisition strategies and enable high quality multi-modal imaging [5], [6], that can be automatically optimized for specific procedure segments [7].

Recent research has been focused on improving intrauterine visualisation; both by improving currently used modalities (for example white light imaging with mosaicing [8], [9]) and introducing new imaging modalities (such as optical ultrasound [10] and photoacoustic imaging [11]). These additional modalities can provide an increased field of view, can yield information below the tissue surface, and can enable visualisation in situations where the amniotic fluid 
is not optically clear. All-optical ultrasound (OpUS), where ultrasound is both generated and received using light [10], is emerging as a versatile modality that is readily miniaturised and hence well-suited to interventional applications. OpUS can provide high resolution imaging from small form factor devices and has recently been demonstrated for real-time in vivo guidance from a surgical needle [12]. Additionally, combination with robotics shows potential for improving control during surgical procedures [4].

This paper presents the design and operation of a rigid, robot-mounted endoscope that for the first time integrates a stereo camera with an OpUS sensor. Through a ROS interface, image acquisition from both modalities is synchonised in realtime during robotic manipulation. After performing a calibration between the sensors and the robot, a tissuemimicking phantom with anatomically realistic vascular structures was imaged using various scan apertures, and the resulting 3D data was collected, processed and displayed in real-time.

\section{INSTRUMENT DESIGN}

\section{A. Mechanical Design}

For this work, a rigid endoscope with a diameter of $5 \mathrm{~mm}$ and a length of $300 \mathrm{~mm}$ was designed and developed. At its proximal end, a custom 3D-printed housing was used to mount the instrument to a robotic manipulator. The endoscope comprised a stainless steel tube $(5 \mathrm{~mm}$ outer diameter, $4.35 \mathrm{~mm}$ inner diameter), and a custom sintered stainless steel endoscope tip. The endoscope tip matched the profile of the instrument shaft, and housed a Naneye Stereo camera (ams AG, Austria), two fibre optic lighting channels, and an $1.1 \mathrm{~mm}$ working channel. The endoscope tip was printed in two sections to allow it to be assembled around the stereo camera. The entire assembled endoscope and endoscope tip can be seen in Fig. 2.

\section{B. Optical Ultrasound}

An all-optical ultrasound probe comprising a fibre optic ultrasound transmitter and a fibre optic ultrasound receiver was fabricated. The ultrasound transmitter comprised a multiwalled carbon nanotube (MWCNT) and polydimethylsiloxane (PDMS) composite [10], [13] coated onto the distal end surface of a $400 \mu \mathrm{m}$ core multimode optical fibre. The ultrasound receiver comprised a plano-concave Fabry-Pérot cavity coated on the distal end surface of a single mode optical fibre (SMF-28) [14]. The two optical fibres were held adjacent, and heat shrink tubing was used to align their distal end surfaces. This pair of optical fibres was housed within an acoustically transparent polymer tube (TPX, outer diameter: $1.2 \mathrm{~mm}$ ) for robustness. The ultrasound probe generated pressures in excess of $2 \mathrm{MPa}$ at a distance of $1.5 \mathrm{~mm}$, with a corresponding $-6 \mathrm{~dB}$ ultrasound bandwidth of $c a$. $30 \mathrm{MHz}$. This OpUS imaging probe achieved an axial imaging resolution of $c a$. $60 \mu \mathrm{m}$ [12].

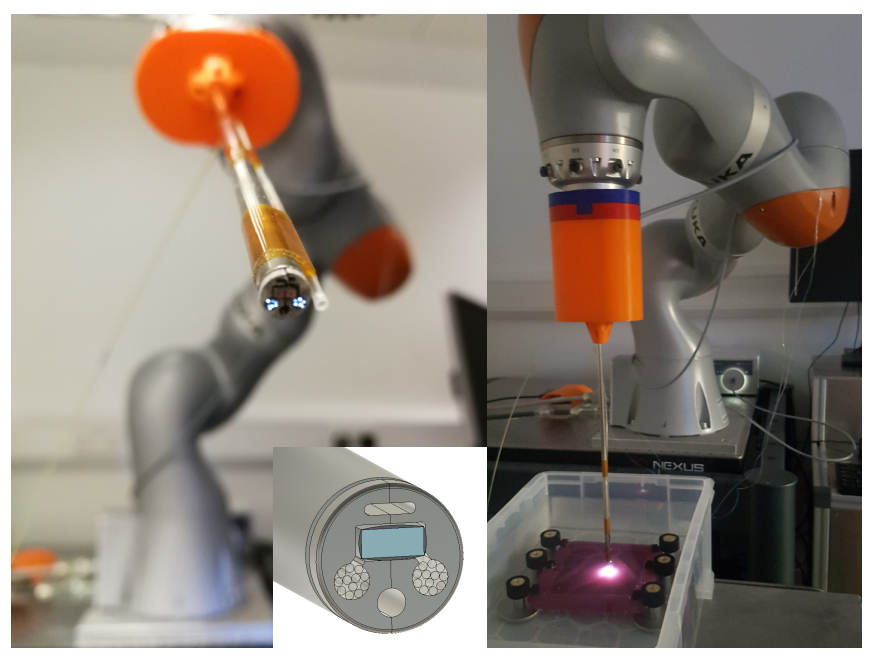

Fig. 2. Left - close-up of assembled endoscope tip showing the Naneye stereo camera, lighting channels, OpUS imaging probe and robotic manipulator. Inset - CAD model of endoscope tip. Right - Photograph of the rigid endoscope positioned above an acoustic placenta phantom (see section IVA).

\section{White Light Imaging}

The Naneye stereo camera has a small footprint measuring $2 \mathrm{~mm} \times 1 \mathrm{~mm}$, while each camera provides a $250 \times 250$ pixel resolution and was constrained through a push fit to the endoscope tip. The fibre optic lighting channels measured $1.1 \mathrm{~mm}$ in diameter, and each channel contained 9 fibres (200 $\mu \mathrm{m}$ core, $500 \mu \mathrm{m}$ coating, $0.5 \mathrm{NA})$. At the proximal end, the 18 optical fibres were fixed within a single Sub Multi Assembly (SMA) connector using epoxy. The resulting bi-furcated fibre bundle was coupled to a standard surgical light source (Cold Light Fountain Power LED 175 SCB, Karl Storz, GmbH, Germany).

\section{Assembly}

In this instance, the OpUS sensor could not be placed within the working channel of the endoscope due to the protective TPX sheath around the sensor. It was therefore mounted to the side of the instrument shaft, thus increasing the overall diameter of the endoscope to $6.2 \mathrm{~mm}$. The assembled endoscope, which can be seen in Fig. 2, was mounted to a KUKA LBR iiwa 14 R820 robotic manipulator (KUKA AG, Germany), which is a 7 DOF manipulator with a payload of $14 \mathrm{~kg}$, a repeatability of $\pm 0.15 \mathrm{~mm}$, and a reach of $800 \mathrm{~mm}$ to enable robotic manipulation.

\section{INTERFACE AND OPERATION}

\section{A. KUKA iiwa ROS Interface}

The KUKA iiwa was controlled through the Fast Robot Interface (FRI), a proprietry application programming interface (API) by KUKA. The FRI is a real-time interface to the robot controller from an external system that utilises universal datagram packet communication. The FRI provides the ability to monitor the current joint positions and torques, and to set the joint position, joint torques and Cartesian wrench at the flange of the robot at $500 \mathrm{~Hz}$ control loop. 
This is implemented as a ROS control hardware interface for controlling the joint positions (joint position interface and joint state interface) [15], which allows joint position, velocity and efforts limits to be set and enforced before being sent to the controller. The Cartesian control and path planning of the manipulator flange is then handled using the Moveit motion planning framework [16], using the TRACIK plugin [17] and the open motion planning library [18] as the inverse kinematics solver and path planner respectively.

\section{B. Camera Interface}

The Naneye cameras were driven through an FPGA controller that polls the two cameras simultaneously to provide synchronised frames. The API for the camera controller is only available for Windows, hence the ROS.Net library was used to interface with the controller on a Windows computer. The resulting images were then published into ROS with the respective camera info [19] at a frame rate of $15 \mathrm{~Hz}$. One example of a pair of synchronised images (corresponding to the left and right camera) is shown in the top of Fig. 3.

\section{US Interface}

The OpUS imaging probe was coupled to a console similar to that previously described [10], [20]. Ultrasound was generated using a Q-switched pulsed laser (DSS-1064 Q, Crylas, Germany) with a wavelength of $1064 \mathrm{~nm}$, a pulse width of $1.5 \mathrm{~ns}$, and a repetition rate of $100 \mathrm{~Hz}$. The pulse energy delivered to the absorbing coating on the ultrasound probe was $20 \mu \mathrm{J}$. The Fabry-Pérot ultrasound receiver was interrogated using a continuous wave tuneable laser (TSL550 , Santec, UK), with a wavelength range $1500-1600 \mathrm{~nm}$, via a circulator. The reflected optical signal from the FabryPérot cavity was measured using a photodetector and digitised at $62.5 \mathrm{MS} / \mathrm{s}$ (M4i.4420-x8, Spectrum, Germany). The low frequency optical signal was used to track the optimum bias point for the sensor. The received ultrasound data was frequency filtered (Butterworth, 4th Order, Bandpass, $3-25 \mathrm{MHz}$ ), had the cross-talk removed, and underwent Hilbert and log transforms for display [10].

The OpUS acquisition was performed using a custom LabVIEW script that streamed both raw and signal-processed A-scans over ROS; an example of a raw A-scan is shown in the bottom of Fig. 3. Using the position and orientation of the endoscope tip obtained from the forward kinematics of the manipulator, the A-scans were drawn in the realworld co-ordinates in real-time. Thus a 3D volume scan could be acquired and displayed in real-time by traversing the endoscope tip across an arbitrary 3D scan pattern. The surface information of the scan was also constructed as a point cloud by finding the maximum intensity point along each A-scan. If this maximum intensity exceeded a certain threshold, it was transformed to the world frame and the colour was mapped to the distance of the point from the endoscope tip. Any outliers of the point cloud were removed using the pcdenoise function in MATLAB (The MathWorks Inc, USA).
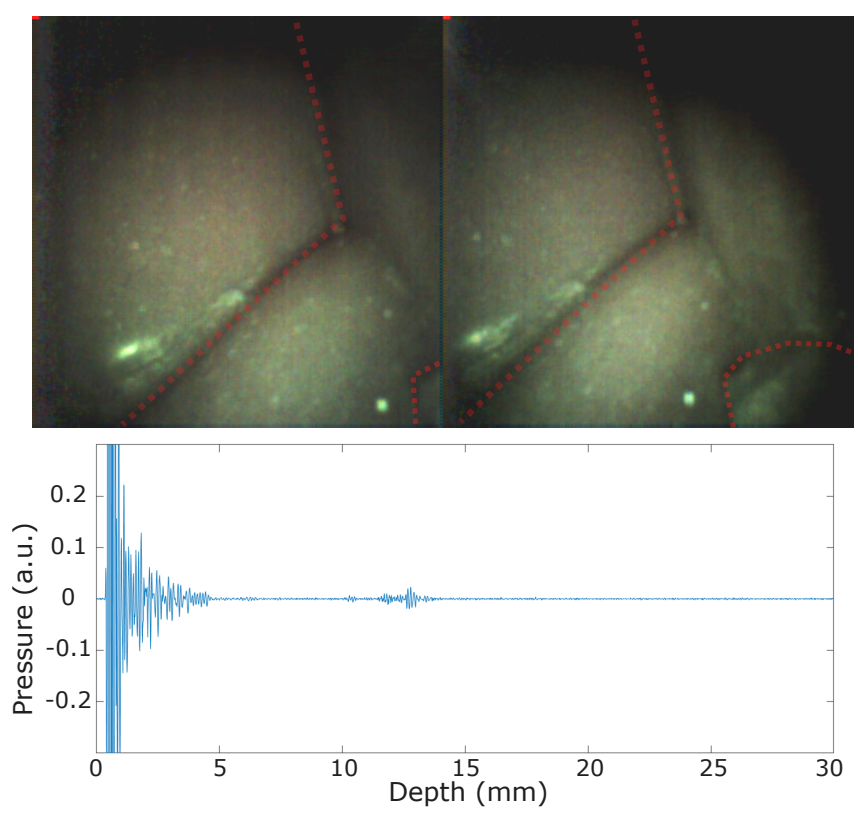

Fig. 3. Top - Example of unprocessed white light images underwater of a placenta phantom obtained from the Naneye Stereo camera using only the integrated lighting channels. Red lines indicate the edges of the placental vessels. Bottom - a raw OpUS A-scan obtained from a tissue-mimicking acoustic placenta phantom. The pulse-echo signals from the phantom can be observed at a depth of approximately $12 \mathrm{~mm}$. The signal observed for depths $<5 \mathrm{~mm}$ is due to direct cross-talk between the fibre-optic ultrasound source and receiver, and is suppressed in subsequent signal processing.

\section{Instrument Control}

When used in minimally invasive procedures, endoscopes are necessarily inserted into the body through the point of incision. In order to minimise stresses on the incision (referred to as the Remote Centre of Motion, or RCM), the endoscope tip position ${ }^{\mathrm{RCM}} \mathbf{T}_{\text {Tip }}$ is constrained to just four degrees of freedom (DOFs) around the RCM: rotation around each of the three axes, and translation along the long axis of the endoscope (referred to as the $Z$ axis). The endoscope tip position can be described as

$$
{ }^{\mathrm{RCM}} \mathbf{T}_{\text {Tip }}=\operatorname{Rot}_{X}(\psi) \operatorname{Rot}_{Y}(\phi) \operatorname{Rot}_{Z}(\gamma) \operatorname{Trans}_{Z}(r)
$$

where $\operatorname{Rot}_{X}(\psi)$ denotes a transform representing a rotation over angle $\psi$ around the $X$ axis, and $\operatorname{Trans}_{Z}(r)$ denotes the transform representing a translation along the $Z$ axis over a distance $r$. Rotations around the $Y$ and $Z$ axes are denoted likewise. Eq. 1 was then analytically inverted to obtain co-ordinates for the four DOFs for a given position and orientation in the $R C M$ frame, Eq. 2.

$$
\begin{aligned}
& \psi=-\sin ^{-1}\left(\frac{y}{\sqrt{\frac{y^{2}+z^{2}}{x^{2}+y^{2}+z^{2}}} \sqrt{x^{2}+y^{2}+z^{2}}}\right) \\
& \phi=\sin ^{-1}\left(\frac{x}{\sqrt{x^{2}+y^{2}+z^{2}}}\right) \\
& r=\sqrt{x^{2}+y^{2}+z^{2}} \\
& \gamma=c
\end{aligned}
$$


Where $x, y$ and $z$ is the desired position along the respective axis and $c$ is the desired orientation about the $Z$ axis. This is implemented within a ROS node which sends the RCM joint co-ordinates through the moveit interface and ensures the RCM constraint is maintained. In addition, this node checks whether the path to the desired position results in collisions within the scene through the scene graph held by moveit.

This ROS node publishes the current joint position, Cartesian position and pose of the RCM, subscribes to the desired joint position and Cartesian position, and advertises a service to set the RCM and to send trajectories in joint or position space. When receiving new commands either as a path or point, the desired position of the tip in joint space is interpolated from the current position. The desired poses are then transformed to the end-effector of the iiwa and the interpolated path is passed to the Cartesian path planner in moveit.

This node is executed alongside the robot interface described in section III-A on an independent computer to the ROS master running a low latency kernel. Distributing the ROS master and robot interface over separate PCs ensured that efficient communication was maintained during FRI execution, while synchronisation with the master computer was achieved through network time protocol synchronisation.

\section{E. Sensor Calibration}

The fetoscope is controlled relative to the tip of the instrument. Therefore to control the robot, the transformation between the robot flange and the tip is needed. The translation of the tip is calibrated to the robot flange through a pivot calibration. The calibration involves pivoting the instrument about the endoscope tip using the point of a cone rigidly fixed to the table as a reference. This is acheived by constraining the tip of the endoscope to a fixed position but free to change orientation while the end-effector of the manipulator is moved into different positions. A sphere is then fitted to the recorded flange positions to find the position of the scope tip. The orientation of the scope tip is taken from the CAD model of the endoscope. The cameras and OpUS are calibrated geometrically from knowledge of the printed endoscope tip and physical measurements.

\section{EXPERIMENTS AND RESULTS}

\section{A. Gel Wax Placenta Phantom}

As imaging target, a placenta phantom was fabricated using gel wax as a tissue-mimicking material. Vascular structures were extracted from a human placenta with 3D drawing software, which were then converted to a 3D printed mould. Solid glass spheres were added to melted gel wax to provide acoustic backscattering. The phantom featured clinically realistic acoustic properties such as propagation speed of sound, echogenicity, and speckle pattern [21]. This phantom was placed into a water bath onto an acrylic sheet and clamped down with metal posts along the side of the phantom, which is shown in Fig. 4.

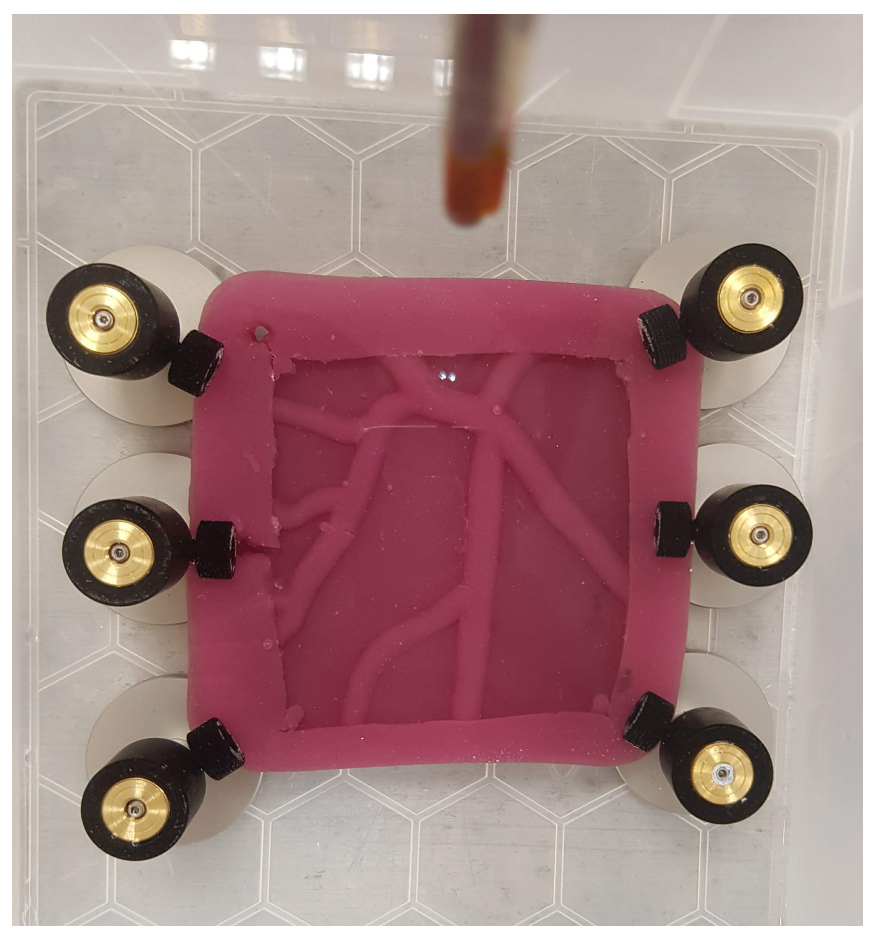

Fig. 4. Photograph of the placenta phantom from above to show the positions of the posts to maintain the position of the phantom.

\section{B. B-Mode Imaging}

The endoscope was scanned across the phantom with a linear trajectory planar to the surface. The trajectory was $40 \mathrm{~mm}$ long and A-scans are collected in $1.0 \mathrm{~mm}$ increments. The A-scans sample points were then transformed into world coordinates and the intensity of points is bound between -50 and $-10 \mathrm{~dB}$ which is then color mapped. The resulting Bscan was acquired in 16 seconds and can be seen in Fig. 5.

\section{Large Scale Scanning}

The phantom was scanned using the endoscope with two independent scanning trajectories: a raster and a spiral trajectory. The RCM was placed approximately in the centre of the phantom and $160.0 \mathrm{~mm}$ above the phantom which corresponds to approximate positioning of instrumentation in clinical use. The raster scan was generated on a plane over the entire $80 \mathrm{~mm} \times 80 \mathrm{~mm}$ area in $1.0 \mathrm{~mm}$ increments at approximately $10 \mathrm{~mm}$ from the phantom. The spiral scan was limited to a total diameter of $80 \mathrm{~mm}$, and comprised 40 cycles around the start point to yield a similar scan density. Due to difficulties in synchronisation between the OpUS acquisition system, robot controller and ROS master, robot motion to each position was followed by a pause of $0.1 \mathrm{~s}$ to ensure a stationary endoscope tip. This start-stop acquisition strategy resulted in a total scanning time of 43 minutes and 45 minutes for the raster and spiral scan, respectively.

The resulting 3D OpUS images are shown in Fig. 6. In these images, the surface vessels on the placenta are clearly visible across the entire imaging aperture. In addition, the supporting acrylic base can be observed at a depth of $35 \mathrm{~mm}$, 

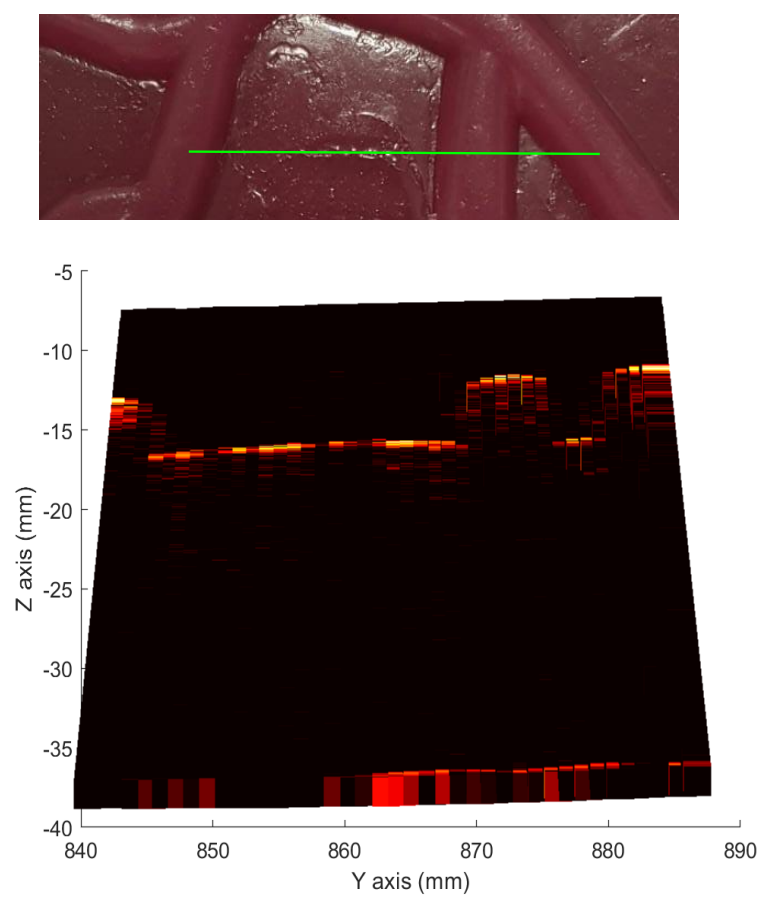

Fig. 5. Top - Top down view of placenta phantom with green line to indicate scan paths. Bottom - Post processed B-scan image of the placenta phantom.

as well as artifacts caused by reflections off the posts used to fix the placenta phantom. The apparent absence of signal along the top edges of the visualisations is likely due to a sub-optimal choice of threshold upon displaying the data; the raw A-scans did indeed capture the placenta phantom surface.

\section{Scan Repeatability and Reliability}

Following the same setup as section IV-C, a $40 \mathrm{~mm} \times 40 \mathrm{~mm}$ raster scan and $40 \mathrm{~mm}$ diameter spiral scan were repeated 10 times. Point clouds of the surface were then generated for each of the scans, and each scan was then registered to the first scan of the same type using the point to point variant of the Iterative Closest Point (ICP) algorithm [22]. The root mean square error, which is the euclidean distance between each point cloud, for each registration is found and the median and quartiles are calculated. The lower, median and upper quartiles are $0.355 \mathrm{~mm}, 0.379 \mathrm{~mm}$ and $0.618 \mathrm{~mm}$ for the raster scan and $0.262 \mathrm{~mm}, 0.265 \mathrm{~mm}, 0.269 \mathrm{~mm}$ for the spiral scan. Each scan was manually segmented to determine the number of outliers from the surface of the placenta phantom (inaccurate data due to noise or other artefacts). The percentage of outliers was $3.12 \%$ and $2.96 \%$ for the raster and spiral scans respectively.

\section{DISCUSSION}

This is the first demonstration of OpUS and optical imaging integrated into an endoscope and controlled by a robotic manipulator. The current instrument has an overall diameter of $6.2 \mathrm{~mm}$, which is larger than the majority of clinically used fetoscopes, which generally do not exceed $4 \mathrm{~mm}$, however current clinical scopes are monocular with a single working channel $<1.0 \mathrm{~mm}$. The OpUS imaging probe provides an A-scan with an imaging depth of $30.0 \mathrm{~mm}$ and an axial resolution of $60 \mu \mathrm{m}$. The imaging probe used had an overall diameter of $1.2 \mathrm{~mm}$ which precluded it from being inserted into the working channel. However, the width at the largest lateral extent of the two optical fibres comprising the ultrasound probe was $<800 \mu \mathrm{m}$, which would allow them to be directly integrated into the working channel of the endoscope. In future iterations of the endoscope design, such integration would provide protection for the ultrasound probe whilst minimizing the total diameter. Additionally, the ultrasound probe used here, which comprised a transmitter based on a $400 \mu \mathrm{m}$ optical fibre, exhibited partial divergence [12]. Thus, the lateral resolution of the system was dependent on the imaging depth and decreased with depth; at its widest the lateral resolution was approximately $2.0 \mathrm{~mm}$. The use of a larger diameter ultrasound transmitter, or one with a concave surface, would lead to improved directivity, and thus improved lateral resolutions [23].

The placenta phantom studied in this work emulated anatomically accurate placental surface vasculature [21]. It was designed to achieve clinically relevant acoustical properties, and through the addition of purple dye the phantom had a passable likeliness with an actual placenta. Using this phantom, OpUS imaging was demonstrated at a penetration depth of up to $20 \mathrm{~mm}$, and the vasculature was clearly visualised and accurately depth-resolved. However, the absence of colour contrast rendered this phantom sub-optimal for white light imaging, which hence revealed only geometry and not function. In future work, a placenta phantom will be developed that has both clinically relevant acoustical and optical properties, which will further increase the value of the combined white light and OpUS imaging performed with the presented rigid endoscope.

One further advantage of the OpUS modality is the ability to integrate complementary modalities by using wavelength selective coatings [20] that selectively absorb or transmit light of different wavelengths. Using such selectively absorbing coatings, the combination of all-optical ultrasound and photoacoustic imaging has recently been demonstrated [20].

The point cloud generated by the OpUS trajectories covers an area of $7127 \mathrm{~mm}^{2}$ and $6474 \mathrm{~mm}^{2}$ for the raster and spiral pattern, respectively. To the authors' knowledge, these images represent the largest OpUS images presented to date. The constructed point clouds clearly shows the vasculature of the phantom; however, the quality of the scan deteriorates with increasing lateral distance from the incision point as the angle between the surface normal and the instrument increases. This is due to the signal processing performed in this work; in future implementations a signal-dependent threshold could be used to improve the homogeneity of the image. In addition, the point clouds presented in this work merely show the voxels exhibiting maximum intensity, resulting in the visualisation of only the surface. In future work, different visualisation techniques (e.g., mapping the signal intensity to the voxel alpha channel) will be implemented to 

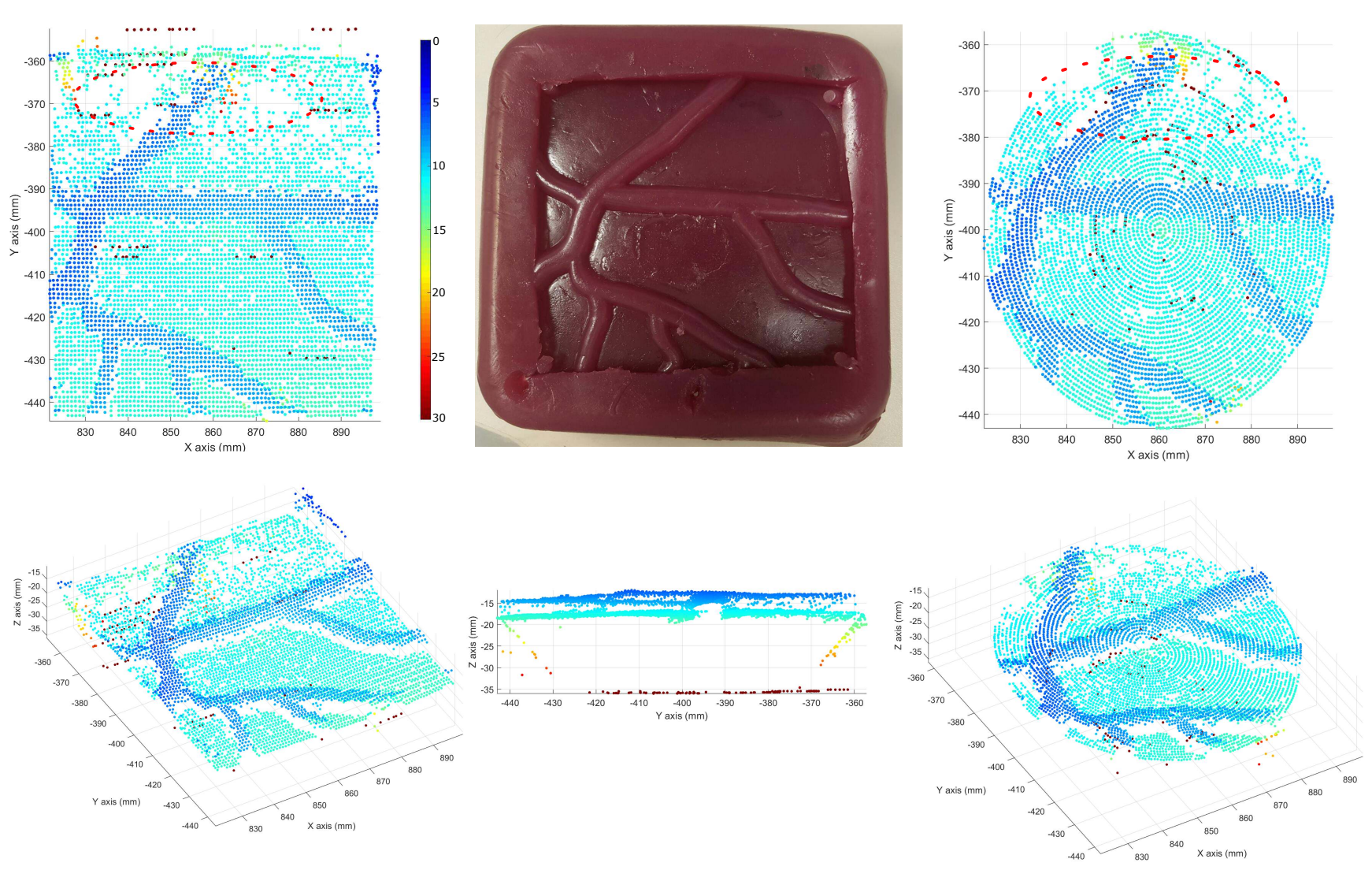

Fig. 6. 3D visualisation of the OpUS images of the placenta phantom acquired through robotic probe manipulation. A photograph of the ultrasound placenta phantom is shown in the centre of the top row. Red dotted lines indicate "holes" in the scan from low signal areas and reflections from the posts holding the phantom down. Left column - 3D OpUS images obtained using a raster scan pattern, in top-down (top) and angled (bottom) view. Right column - 3D OpUS images obtained using a spiral scan pattern. Bottom centre - side-view of the 3D OpUS image obtained using a spiral scan pattern. In each image the distance of the points from the endoscope tip are colour-encoded using the colorbar (in mm) displayed in the top left panel.

enable sub-surface visualisations as well.

The repeatability assessment of the scans demonstrates the potential for the endoscope to be returned to previously scanned positions after the point cloud has been constructed. This could be used in surgery to assist in enforcing the distance and angle constraints for laser coagulation, and after coagulation it could be used to assess the quality of the coagulation.

The current system requires a stop in the motion to allow for ultrasound data to be synchronised with the step position, resulting in an acquisition rate of approximately 2.5 points per second. However, the OpUS and ROS interfaces allow for data transmission at a rate of approximately $60 \mathrm{~A}$-scans per second. In future work, the acquisition will be accelerated by synchronising the acquisition PCs or providing timestamps with the ultrasound data. This change would allow for a smooth continuous motion during acquisition, and thus faster acquisition speeds. Within the current robot control strategy, this would result in scans of equal area and density shown acquired in 2 minutes 35 seconds and 4 minutes 5 seconds for the raster and spiral scans, respectively, which is more conducive to clinical timescales.

\section{CONCLUSIONS}

This paper has presented the design, control, and operation of a multimodal robotic endoscope with integrated white light optical and OpUS imaging. The endoscope consists of a miniature stereo camera, fibre optic light channels and an optical ultrasound sensor within a $6.2 \mathrm{~mm}$ overall diameter. The instrument is manipulated by a KUKA LBR iiwa R820 arm, constrained through software to a remote centre of motion.

The endoscope is demonstrated through generating scanning paths over a gel wax placenta phantom. A-scans acquired by the OpUS sensor are processed into B-scans and 3d surfaces using the kinematics of the endoscope. The vessels can be clearly identified from the main body of the placenta. To the authors' knowledge, the OpUS scans presented in Fig. 6 are the largest presented in literature.

Future work will focus on minaturisation of the endoscope while introducing an internal channel for the OpUS sensor and therapeutic laser light delivery. In addition, the integration between the imaging sensors and robot manipulator will be utilised to constrain the robot to the surgical scene and to introduce adaptive scanning trajectories. 


\section{REFERENCES}

[1] P. Klaritsch, K. Albert, T. Van Mieghem, L. Gucciardo, E. Done', B. Bynens, and J. Deprest, "Instrumental requirements for minimal invasive fetal surgery.," BJOG, vol. 116, pp. 188-97, January 2009.

[2] G. E. Chalouhi, M. Essaoui, J. Stirnemann, T. Quibel, B. Deloison, L. Salomon, and Y. Ville, "Laser therapy for twin-to-twin transfusion syndrome (TTTS).," Prenat. Diagn., vol. 31, pp. 637-46, July 2011.

[3] G. Dwyer, F. Chadebecq, M. T. Amo, C. Bergeles, E. Maneas, V. Pawar, E. V. Poorten, J. Deprest, S. Ourselin, P. De Coppi, T. Vercauteren, and D. Stoyanov, "A Continuum Robot and Control Interface for Surgical Assist in Fetoscopic Interventions," IEEE Robot. Autom. Lett., vol. 2, pp. 1656-1663, July 2017.

[4] C. Gruijthuijsen, R. Colchester, A. Devreker, A. Javaux, E. Maneas, S. Noimark, W. Xia, D. Stoyanov, D. Reynaerts, J. Deprest, S. Ourselin, A. Desjardins, T. Vercauteren, and E. V. Poorten, "Haptic Guidance Based on All-Optical Ultrasound Distance Sensing for Safer Minimally Invasive Fetal Surgery," J. Med. Robot. Res., p. 1841001, March 2018.

[5] M. S. Erden, B. Rosa, J. Szewczyk, and G. Morel, "Understanding soft-tissue behavior for application to microlaparoscopic surface scan.," IEEE Trans. Biomed. Eng., vol. 60, pp. 1059-68, April 2013.

[6] P. Wisanuvej, P. Giataganas, K. Leibrandt, J. Liu, M. Hughes, and G.Z. Yang, "Three-dimensional robotic-assisted endomicroscopy with a force adaptive robotic arm," in 2017 IEEE Int. Conf. Robot. Autom., pp. 2379-2384, IEEE, May 2017.

[7] F. Vasconcelos, P. Brandão, T. Vercauteren, S. Ourselin, J. Deprest, D. Peebles, and D. Stoyanov, "Towards computer-assisted TTTS: Laser ablation detection for workflow segmentation from fetoscopic video," Int. J. Comput. Assist. Radiol. Surg., vol. 13, pp. 1661-1670, October 2018.

[8] M. Tella, P. Daga, F. Chadebecq, S. Thompson, D. I. Shakir, G. Dwyer, W. Ruwan, J. Deprest, D. Stoyanov, T. Vercauteren, and S. Ourselin, "A combined EM and visual tracking probabilistic model for robust mosaicking of fetoscopic videos," IEEE Proc. 7th Int. Work. Biomed. Image Regist., 2016.

[9] P. Daga, F. Chadebecq, D. Shakir, L. C. Garcia-Peraza Herrera, M. Tella, G. Dwyer, A. L. David, J. Deprest, D. Stoyanov, T. Vercauteren, and S. Ourselin, "Real-time mosaicing of fetoscopic videos using SIFT," SPIE Med. Imaging Proc., vol. 9786, pp. 1-7, 2015

[10] R. J. Colchester, E. Z. Zhang, C. A. Mosse, P. C. Beard, I. Papakonstantinou, and A. E. Desjardins, "Broadband miniature optical ultrasound probe for high resolution vascular tissue imaging," Biomed. Opt. Express, vol. 6, p. 1502, April 2015.

[11] R. Ansari, E. Zhang, A. E. Desjardins, and P. C. Beard, "All-optical endoscopic probe for high resolution 3D photoacoustic tomography," p. $100641 \mathrm{~W}$, March 2017.
[12] M. C. Finlay, C. A. Mosse, R. J. Colchester, S. Noimark, E. Z. Zhang, S. Ourselin, P. C. Beard, R. J. Schilling, I. P. Parkin, I. Papakonstantinou, and A. E. Desjardins, "Through-needle all-optical ultrasound imaging in vivo: a preclinical swine study," Light Sci. Appl., vol. 6 , p. e17103, December 2017.

[13] S. Noimark, R. J. Colchester, B. J. Blackburn, E. Z. Zhang, E. J. Alles, S. Ourselin, P. C. Beard, I. Papakonstantinou, I. P. Parkin, and A. E. Desjardins, "Carbon-Nanotube-PDMS Composite Coatings on Optical Fibers for All-Optical Ultrasound Imaging," Adv. Funct. Mater, vol. 26, pp. 8390-8396, December 2016.

[14] J. A. Guggenheim, J. Li, T. J. Allen, R. J. Colchester, S. Noimark, O. Ogunlade, I. P. Parkin, I. Papakonstantinou, A. E. Desjardins, E. Z. Zhang, and P. C. Beard, "Ultrasensitive plano-concave optical microresonators for ultrasound sensing," Nat. Photonics, vol. 11, pp. 714-719, November 2017.

[15] S. Chitta, E. Marder-Eppstein, W. Meeussen, V. Pradeep, A. Rodríguez Tsouroukdissian, J. Bohren, D. Coleman, B. Magyar, G. Raiola M. Lüdtke, and E. Fernandez Perdomo, "ros_control: A generic and simple control framework for ROS," J. Open Source Softw., vol. 2 p. 456, December 2017.

[16] S. Chitta and I. A. Sucan, "Moveit!." Available at http://moveit.ros.org/.

[17] P. Beeson and B. Ames, " $\{$ TRAC-IK $\}$ : An Open-Source Library for Improved Solving of Generic Inverse Kinematics," in Proc. IEEE RAS Humanoids Conf., (Seoul, Korea), November 2015.

[18] I. A. Şucan, M. Moll, and L. E. Kavraki, "The Open Motion Planning Library," IEEE Robotics \& Automation Magazine, vol. 19, pp. 72-82, December 2012. http://ompl.kavrakilab.org.

[19] E. McCann, "ROS.NET," 2011. Available at https://github.com/umlrobotics/ROS.NET.

[20] S. Noimark, R. J. Colchester, R. K. Poduval, E. Maneas, E. J. Alles, T. Zhao, E. Z. Zhang, M. Ashworth, E. Tsolaki, A. H. Chester, N. Latif S. Bertazzo, A. L. David, S. Ourselin, P. C. Beard, I. P. Parkin, I. Papakonstantinou, and A. E. Desjardins, "Polydimethylsiloxane Composites for Optical Ultrasound Generation and Multimodality Imaging," Adv. Funct. Mater. vol. 28, p. 1704919, February 2018

[21] E. Maneas, W. Xia, D. I. Nikitichev, B. Daher, M. Manimaran, R. Y. J. Wong, C.-W. Chang, B. Rahmani, C. Capelli, S. Schievano, G. Burriesci, S. Ourselin, A. L. David, M. C. Finlay, S. J. West, T. Vercauteren, and A. E. Desjardins, "Anatomically realistic ultrasound phantoms using gel wax with 3D printed moulds," Phys. Med. Biol., vol. 63 , p. 015033, January 2018.

[22] P. J. Besl and N. D. McKay, "A method for registration of 3-d shapes," IEEE Transactions on Pattern Analysis and Machine Intelligence, vol. 14, pp. 239-256, February 1992.

[23] E. J. Alles, S. Noimark, E. Zhang, P. C. Beard, and A. E. Desjardins, "Pencil beam all-optical ultrasound imaging," Biomed. Opt. Express, vol. 7, p. 3696, September 2016. 